\title{
Protein biomarkers in blood reflect the interrelationships between stroke outcome, inflammation, coagulation, adhesion, senescence and cancer
}

\author{
Georg Fuellen*1, Jan Böhmert², Larissa Henze ${ }^{3}$, Daniel Palmer ${ }^{1}$ Uwe Walter ${ }^{2}$, Axel Kowald¹ \\ 1 Institute for Biostatistics and Informatics in Medicine and Ageing Research, Rostock University Medical Center and \\ Centre for Transdisciplinary Neurosciences Rostock and Research Focus Oncology, Rostock and Ageing of \\ Individuals and Society, Interdisciplinary Faculty, Rostock University, Rostock, Germany \\ 2 Department of Neurology, Rostock University Medical Center and Centre for Transdisciplinary Neurosciences \\ Rostock, Rostock, Germany \\ 3 Department of Medicine, Clinic III, Hematology, Oncology, Palliative Medicine, Rostock University Medical Center \\ and Research Focus Oncology, Rostock, Germany \\ *Corresponding Authors, fuellen@uni-rostock.de; axel.kowald@uni-rostock.de.
}

Keywords: stroke, cellular senescence, coagulation, adhesion

\begin{abstract}
The most important predictors for outcomes after ischemic stroke, that is, for health deterioration and death, are chronological age and stroke severity; gender, genetics and environmental factors also play a role. Of all these, only the latter can be influenced after the event, even though recurrent stroke may be prevented by antiaggregant/anticoagulant therapy, angioplasty of high-grade stenoses, and treatment of cardiovascular risk factors. Moreover, blood cell composition and protein biomarkers such as C-reactive protein or interleukins in serum/plasma are frequently considered as biomarkers of outcome, and they are connected to underlying molecular mechanisms such as inflammation, hypercoagulation, and cellular senescence. We surveyed protein biomarkers that were reported to be predictive for outcome after ischemic stroke, specifically considering biomarkers that predict long-term outcome ( $\geq 3$ months) and that are measured over the first days following the event. We classified the protein biomarkers as immune $\square$ inflammatory, coagulation-related, and adhesion-related biomarkers. Some of these biomarkers are closely related to cellular senescence and, in particular, to the inflammatory processes that can be triggered by senescent cells. Moreover, the processes that underlie inflammation, hypercoagulation and cellular senescence connect stroke to cancer, and biomarkers of cancer-associated thromboembolism, as well as of sarcopenia, overlap strongly with the biomarkers discussed here. Finally, we demonstrate that many of the outcomepredicting protein biomarkers form a close-meshed functional interaction network, suggesting that the outcome after stroke is partially determined by an interplay of the molecular processes relating to inflammation, coagulation, cell adhesion and cellular senescence.
\end{abstract}




\section{Introduction}

Stroke biomarkers, specifically predictors of outcome after ischemic stroke (stroke, for short), are an active and important area of research. The prevalence, loss of quality-of-life, reduction in life expectancy, and the limited treatment options after the event, are all responsible for its high socio-economic burden (Uivarosan et al., 2020). Traditionally, chronological age and stroke severity were investigated alongside gender, genetics and environmental factors such as smoking (Donkor, 2018). For primary prevention in particular, further environmental factors such as diet or lifestyle are important (O'Donnell et al., 2016). In terms of secondary prevention, antiaggregant/anticoagulant therapy, angioplasty of high-grade stenoses of cerebral arteries, and medical treatment of cardiovascular risk factors such as hyperlipidemia, diabetes and arterial hypertension are administered, in particular if risk factors such as arteriosclerotic stenosis, atrial fibrillation, hypertension or dyslipidemia are present (Hankey, 2014). Tertiary prevention, e.g. of dementia and frailty, includes cognitive and somatic rehabilitation (Draaisma et al., 2020).

To tailor preventive strategies to the patient, biomarkers are essential. Optimally, these predict future disease, dysfunction or death, provide hints at underlying causal mechanisms, and predict the results of interventions. Biological age is a general predictor of (comorbid) disease and dysfunction (Fuellen et al., 2020). Estimated by an epigenetic (DNA methylation) assay, it contributes to outcome prediction after stroke (Soriano-Tarraga et al., 2021), and patients with a high biological age may receive more frequent care. Other predictors are based on gene expression (Sykes et al., 2021) and protein abundance (Montellano et al., 2021); analysis of proteins is generally established. Proteins are expected to be involved directly in the molecular processes that influence the outcome, and they are informative about molecular mechanisms and intervention success rates, and therefore we focus on those in this review. We only consider other biomarkers if these are connected to proteins, by forming biomarker signatures with them. The mechanistic role that can be ascribed to proteins allows two important steps towards a better understanding of their function as biomarkers for the natural history of the progression of disease: First, we can assign the proteins to specific molecular/cellular processes deemed important for the health deterioration observed after stroke. Here, we follow up upon the classification by Lehmann et al. (2021), considering immune $\square$ inflammatory, coagulation-related, and adhesion-related proteins. Second, we can generate a protein interaction network from the proteins that describes their molecular interrelationships. Here, we employ the STRING (Jensen et al., 2009) database, and the resulting functional interaction network describes how the proteins, and therefore the molecular/cellular processes they participate in, are intertwined.

\section{Data collection and tabulation: Blood biomarkers of stroke relate to immunity, inflammation, coagulation, and adhesion}

For this narrative review, we surveyed the literature on protein biomarkers for human ischemic stroke outcome prediction in adults, published from September 1, 2018 until July 30, 2021. We included reviews, specifically to cover the time before 2018 , as well as the primary literature, to cover more recent developments. All papers had to include protein biomarkers; we also list biomarkers of other types if these are mentioned alongside the proteins. All biomarkers had to 
predict long-term (chronic, $\geq 3$ months) outcome; their assignment to the categories of immune $\square$ inflammation-related, coagulation-related, and adhesion-related was then based on the papers that report the markers, following the scheme by Lehmann et al. (2021). Finally, we used the STRING database (Jensen et al., 2009) to exemplify a functional interaction network in which most of the protein biomarkers are involved. More specifically, we took all proteins from the table, submitted them to STRING, and captured the resulting network, as well as the gene ontology biological process annotation provided by STRING.

We first considered systematic reviews, from which we only transcribed the high-quality biomarkers into Table 1, that is, the biomarkers most consistently associated with outcome and (if available) with an added value compared to clinical routine (that is, compared to standard clinical and demographic markers that are routinely measured). We found no meta-analyses; this observation may be explained by the heterogeneity of the existing studies, according to Montellano et al. (2021). In fact, the review by Montellano et al. is the most exhaustive recent systematic review, based on 291 studies, screened until August 2018, including biomarkers measured up to 7 days post-stroke, and predicting outcomes thereafter. As the abstract states, "natriuretic peptides, copeptin, procalcitonin, mannose-binding lectin, adipocyte fatty acidbinding protein, and cortisol were the biomarkers most consistently associated with poor outcome in higher-quality studies showing an incremental value over established prognostic factors", where established factors refer clinical routine. These high-quality biomarkers we report in Table 1 (top), together with a few "atherogenesis" biomarkers highlighted by the same authors, reported by higher-quality studies to be associated with poor outcome (but with no explicit reference to any incremental/added value). The authors summarize the roles of the biomarkers as "inflammation, atherogenesis, and stress response", which overlaps with our classification of immune $\square$ inflammation-related, coagulation-related, and adhesion-related biomarkers. Notably, they also report that CRP, TNFa and two cellular measurements (NLR (neutrophil-lymphocyte ratio) and WBC (white-blood cell count)) only feature inconsistent associations, while IL-6 and MMP-9 are only supported by one or two higher-quality studies of sufficient size, so these biomarkers could not meet the "most consistently associated in higherquality studies showing an incremental value" criterion.

Donkel et al. (2019) provide another systematic review, based on 41 studies, screened until June 2018, with a focus on biomarkers of the hemostatic system (measured up until $72 \mathrm{~h}$ postevent) as predictors, and disability (not considering death) as the outcome, and we list their results in Table 1 as well. More specifically, we went through their main table and transcribed biomarkers with evidence from more than one study, and where the majority of these studies had to demonstrate an association with outcome, but not necessarily as an association with an added value (i.e., the association did not have to be demonstrated in, e.g., a multivariable regression analysis). Study quality was reported in the supplement, but all studies that met the inclusion criteria were included, irrespective of the quality score. The authors only consider biomarkers related to coagulation in the first place, without any further classification. Lai et al. (2019) also provide a systematic review, based on 18 studies, screened until September 2018, with a focus on biomarkers measured around $24 \mathrm{~h}$ post-event, as predictors of (recovery of) physical function (not considering death) as the outcome; their biomarkers were included in Table 1 if they were reported to be "[c]onsistently [...] found to be robust predictors of long-term functional outcome in ischemic stroke" (Lai et al., 2019). Study quality is assessed by Lai et al. (2019), but not used further in their analysis. The biomarkers are classified as related to immune response, lipids/metabolism 
(no protein markers), neuronal function and blood vessel/circulation, again closely overlapping with our classification.

Next, we screened the primary literature from September 1, 2018 onwards, from which we transcribed biomarkers even if their added value was not established, into Table 1 (bottom). As noted by Montellano et al. (2021), few original studies consider the added value of the biomarkers they investigate, by reporting prediction models that consider clinical routine data as well (often, not even age, nor stroke severity are considered), and it appears that the situation has not changed much since the August 2018 cutoff of Montellano et al. (2021). Also, we did not formally assess study quality ourselves, and the overall number of participants is merely noted (though it is larger than 100 in all cases). Nevertheless, more recent studies usually feature more participants and, often, higher methodological quality, justifying the less strict criteria we imposed upon the recent primary literature.

In particular, from the study by Reiche et al. (2019), using the modified Rankin scale (mRS) as outcome, we only considered the predictor (not the association) analyses, based on logistic regression. We ignored the two composite scores reflecting inflammatory indices. The biomarkers were characterized as "immune-inflammatory, oxidative stress and biochemical". From the follow-up study by Alfieri et al. (2020), using 3-month mortality as outcome, we again only employed the predictor (not the association) analyses, although these included all variables without any adjustment, employing logistic regression. Among the four regression analyses presented, we took the one considering all biomarkers and the demographic/clinical data. Similarly to the first study, biomarkers were characterized as related to "immuneinflammatory, metabolic, oxidative, and nitrosative stress". In a third study by an overlapping set of authors, (Lehmann et al., 2021), using 1-year mortality as outcome, the authors calculate predictors by logistic regression, neural network and support-vector-machine, and we took the consensus biomarkers as reported in the discussion section of the paper, considering these to be the most robust predictors. As already noted, biomarkers were classified as belonging to "immune $\square$ inflammatory, coagulation and adhesion" (carotid intima-media thickness was not part of the consensus), and we adopted this scheme as the blueprint for our review, since these also fit well with the biological processes ascribed to the biomarkers found in the three systematic reviews.

We found a few more recent studies concerned with predicting outcome after stroke. Li et al. (2021) confirmed Lp-PLA2, which was already noted by Montellano et al. (2021). Finally, Li et al. (2019) investigated cytokine profiles, and found IL5 and IL6 as independent predictors of outcome.

Table 1. Blood-based biomarkers that predict stroke outcomes. Stringent criteria were applied to biomarkers reported in systematic reviews. HUGO gene names are given in parentheses where applicable. The color code of the biomarkers is red (immune-inflammatory), blue (coagulation-related) and magenta (adhesion-related). Non-protein biomarkers are given in italics. mRS: modified Rankin scale.

\begin{tabular}{|l|l|l|l|l|l|l|}
\hline Referen & Biomarkers & Time & Other & Method / & Outcome & Number of \\
\hline
\end{tabular}




\begin{tabular}{|c|c|c|c|c|c|c|}
\hline ce & & $\begin{array}{l}\text { measured, } \\
\text { post-event }\end{array}$ & $\begin{array}{c}\text { clinical } \\
\text { variables } \\
\text { considered / } \\
\text { adjusted for }\end{array}$ & $\begin{array}{l}\text { prediction } \\
\text { algorithm }\end{array}$ & predicted & $\begin{array}{c}\text { studies or } \\
\text { partici- } \\
\text { pants }\end{array}$ \\
\hline \multicolumn{7}{|c|}{ Stroke outcomes, systematic reviews covering the time before September 2018} \\
\hline $\begin{array}{l}\text { Montella } \\
\text { no et al. } \\
(2021)\end{array}$ & $\begin{array}{l}\text { natriuretic peptides, } \\
\text { copeptin, cortisol; } \\
\text { procalcitonin; } \\
\text { mannose-binding } \\
\text { lectin (MBL2); } \\
\text { adipocyte fatty acid- } \\
\text { binding protein } \\
\text { (FABP4); free fatty } \\
\text { acids, lipoprotein(a) } \\
\text { (LPA), lipoprotein- } \\
\text { associated } \\
\text { phospholipase } \\
\text { A2 (PLA2G7), } \\
\text { osteoprotegerin } \\
\text { (TNFRSF11B), } \\
\text { homocysteine; d- } \\
\text { dimers }\end{array}$ & $\begin{array}{l}\text { up until } 7 \\
\text { days }\end{array}$ & $\begin{array}{l}\text { age and } \\
\text { severity (in } \\
\text { the majority } \\
\text { of studies); } \\
\text { etiology in a } \\
\text { minority of } \\
\text { studies }\end{array}$ & $\begin{array}{l}\text { systematic } \\
\text { review }\end{array}$ & $\begin{array}{l}\text { functional } \\
\text { outcome or } \\
\text { mortality } 7 \\
\text { days post- } \\
\text { event or later }\end{array}$ & 291 studies \\
\hline $\begin{array}{l}\text { Donkel } \\
\text { et al. } \\
\text { (2019) }\end{array}$ & $\begin{array}{l}\text { Fibrinogen (FGA), } \\
\text { prothrombin } \\
\text { fragment } 1+2, d- \\
\text { dimers, PAI-1 } \\
\text { (SERPINE1) }\end{array}$ & $\begin{array}{l}\text { up until } \\
72 \mathrm{~h}\end{array}$ & $\begin{array}{l}\text { various, } \\
\text { depending } \\
\text { on study }\end{array}$ & $\begin{array}{l}\text { systematic } \\
\text { review }\end{array}$ & $\begin{array}{l}\text { disability } \\
\text { score } \\
\text { (Barthel } \\
\text { Index or } \\
\text { mRS) }\end{array}$ & 41 studies \\
\hline $\begin{array}{l}\text { Lai et al. } \\
(2019)\end{array}$ & $\begin{array}{l}\text { CRP, IL6, TNFa } \\
\text { (TNF) }\end{array}$ & $\begin{array}{l}\text { around } \\
24 \mathrm{~h}\end{array}$ & $\begin{array}{l}\text { various, } \\
\text { depending } \\
\text { on study }\end{array}$ & $\begin{array}{l}\text { systematic } \\
\text { review }\end{array}$ & $\begin{array}{l}\text { physical } \\
\text { outcome }\end{array}$ & 18 studies \\
\hline \multicolumn{7}{|c|}{ Stroke outcomes, original papers since September 2018} \\
\hline $\begin{array}{l}\text { Lehman } \\
\mathrm{n} \text { et al. } \\
(2021)\end{array}$ & $\begin{array}{l}\text { IL6, TNFa (TNF), } \\
\text { TGF- } 1 \text { 1 (TGBB1), } \\
\text { Protein S (PROS1), } \\
\text { Protein C (PROC), } \\
\text { VWF, platelet count, } \\
\text { VCAM1, E-selectin } \\
\text { (SELE) }\end{array}$ & within $24 \mathrm{~h}$ & $\begin{array}{l}\text { carotid } \\
\text { intima-media } \\
\text { thickness } \\
\text { (cIMT) }\end{array}$ & $\begin{array}{l}\text { consensus } \\
\text { of logistic } \\
\text { regression, } \\
\text { neural } \\
\text { network } \\
\text { and } \\
\text { support- } \\
\text { vector- } \\
\text { machine }\end{array}$ & $\begin{array}{l}\text { mortality at } 1 \\
\text { year }\end{array}$ & $\begin{array}{l}103 \\
\text { patients }\end{array}$ \\
\hline $\begin{array}{l}\text { Alfieri et } \\
\text { al. } \\
(2020)\end{array}$ & $\begin{array}{l}\text { systolic blood } \\
\text { pressure, glucose, } \\
\text { NOx, hydroperoxides, } \\
\text { IL6, WBC, 25(OH)D }\end{array}$ & within $24 \mathrm{~h}$ & $\begin{array}{l}\text { all variables } \\
\text { included; } \\
\text { sex is } \\
\text { predictive as } \\
\text { well }\end{array}$ & $\begin{array}{l}\text { binary } \\
\text { logistic } \\
\text { regression }\end{array}$ & $\begin{array}{l}\text { mRS at } 3 \\
\text { months }\end{array}$ & $\begin{array}{l}176 \\
\text { patients } \\
176 \\
\text { controls }\end{array}$ \\
\hline
\end{tabular}




\begin{tabular}{|c|c|c|c|c|c|c|}
\hline $\begin{array}{l}\text { Reiche } \\
\text { et al. } \\
(2019)\end{array}$ & $\begin{array}{l}\text { IL6, ferritin (FTH1), } \\
\text { glucose, } \\
\text { lipid hydroperoxides, } \\
25(\mathrm{OH}) D\end{array}$ & within $24 \mathrm{~h}$ & $\begin{array}{l}\text { not } \\
\text { considered }\end{array}$ & $\begin{array}{l}\text { binary } \\
\text { logistic } \\
\text { regression }\end{array}$ & $\begin{array}{l}\text { mortality at } 3 \\
\text { months }\end{array}$ & $\begin{array}{l}145 \\
\text { patients } \\
176 \\
\text { controls }\end{array}$ \\
\hline $\begin{array}{l}\text { Li et al. } \\
\text { (2021) }\end{array}$ & Lp-PLA2 (PLA2G7) & & & & $\begin{array}{l}\text { stroke } \\
\text { recurrence }\end{array}$ & $\begin{array}{l}251 \text { stroke } \\
\text { patients } \\
100 \\
\text { controls }\end{array}$ \\
\hline $\begin{array}{l}\text { Li et al. } \\
\text { (2019) }\end{array}$ & $\begin{array}{l}\text { IL5, IL6 (additionally, } \\
\text { without adjustment: } \\
\text { IL1 } \beta, \text { IL4, IL7, IL9, } \\
\text { IL10, IL15, G-CSF, } \\
\text { GM-CSF) }\end{array}$ & within $24 \mathrm{~h}$ & $\begin{array}{l}\text { gender, age, } \\
\text { smoking, } \\
\text { alcohol } \\
\text { abuse, } \\
\text { hypertension, } \\
\text { diabetes, } \\
\text { hyperlipidemi } \\
\text { a, } \\
\text { cardiovascul } \\
\text { ar diseases, } \\
\text { blood } \\
\text { pressure }\end{array}$ & $\begin{array}{l}\text { multivariate } \\
\text { logistic } \\
\text { regression } \\
\text { analysis }\end{array}$ & $\begin{array}{l}\mathrm{mRS} \text { at } 3 \\
\text { months }\end{array}$ & $\begin{array}{l}180 \\
\text { patients }\end{array}$ \\
\hline
\end{tabular}

\section{Interaction network and gene ontology analysis of blood biomarkers of stroke corroborate their known roles}

As described, the protein biomarkers for predicting outcome after ischemic stroke that we tabulated in this review (Table 1) can be assigned to the three classes of immune $\square$ inflammatory, coagulation-related, and adhesion-related proteins, following the scheme of Lehmann et al. (2021). The STRING functional interaction network assembled from these proteins (Fig. 1) groups them in the same way, placing immune $\square$ inflammatory proteins into the center of the network. PAI-1/SERPINE1 is given the role of a hub, reflecting its involvement in inflammation as well as coagulation (Hisada \& Mackman, 2017; Valenzuela et al., 2017). The inflammation-associated proteins IL6, CRP, TNF and TGFB1 are also given a central role. Non-protein biomarkers mentioned alongside the proteins in Table 1 refer to blood cell composition (platelets and white blood cells), glucose and lipids (free fatty acids and lipid hydroperoxides), remnants of coagulation processes (d-dimers, prothrombin fragment) (Friedmann et al., 2019), as well as cardiac markers (natriuretic peptides, copeptin, blood pressure, relating to endothelial/vascular dysfunction) and general stress/health determinants (procalcitonin, cortisol, vitamin D). Most of these non-protein biomarkers are therefore also closely related to inflammation/immunity and to coagulation, and as future work we could envision a network that includes both protein and non-protein biomarkers, which may be based on publication-derived co-mentionings, or correlation data from longitudinal studies.

Our finding that ferritin (FTH1) was not connected in the network of blood-based biomarkers predictive of stroke outcomes deserves comment. In recent studies, ferritin in acute stroke patients was related to baseline rather than long-term disability (Reiche et al., 2019; Alfieri et al., 2020; Garg et al., 2020). It has been shown in the animal model that increased body iron 
indicated by ferritin worsens ischemic damage induced by transient ischemia and early reperfusion (Garcia-Yebenes et al., 2012). Also in humans, increased serum ferritin was related to poor outcome after thrombolytic therapy in acute stroke due to early hemorrhagic transformation and severe brain edema (Millan et al., 2007). Extracellular ferritin iron exacerbates the neurotoxic effect induced by glutamate excitotoxicity which plays a crucial role in acute brain ischemia (Millan et al., 2008; Gamez et al., 2021). Together with our STRING network analysis finding, we propose that ferritin mainly influences acute stroke severity, which is already reflected by clinical scores such as the NIHSS, and therefore does not add value in the prediction of long-term outcome.

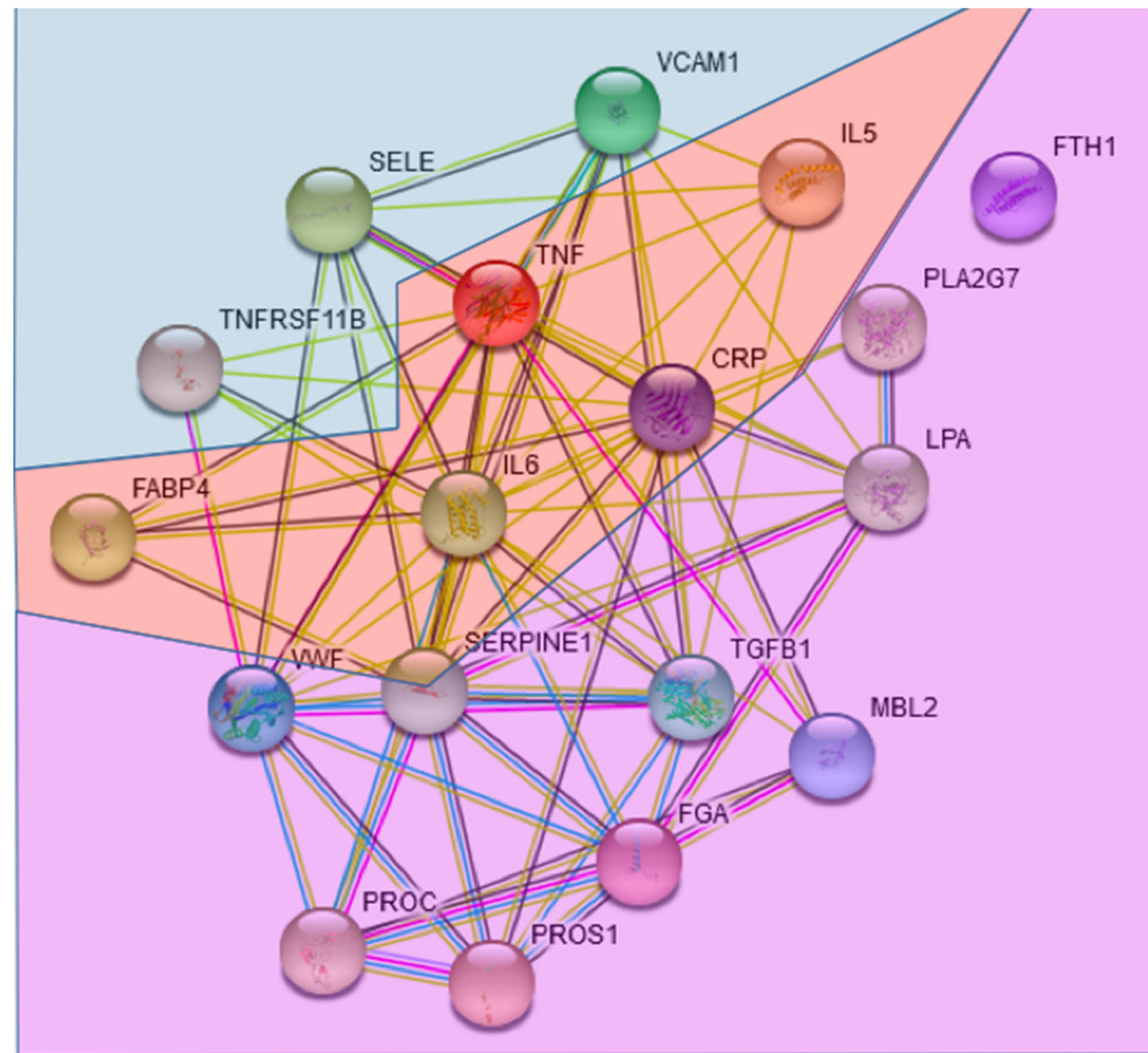

Fig. 1: STRING network of the proteins in Table 1. The three adhesion-related biomarkers are positioned at the top; the coagulation-related biomarkers are positioned at the bottom. The immune-inflammation biomarkers are found in the middle of the network (pale red). Even though TNFRSF11B is not easy to classify, it increases leukocyte adhesion to endothelial cells (Zauli et al., 2007), rendering it an adhesion-related protein. Only FTH is not connected in the STRING-based network, using default parameters.

Table 2: Gene ontology biological process enrichment (first 50 terms) provided by STRING for the network of Fig. 1. The color code of the biomarkers is red (immune-inflammatory), blue (coagulation-related) and magenta (adhesion-related). The pale red color refers to immuneinflammatory processes in a wider sense. FDR: false discovery rate. 


\begin{tabular}{|c|c|c|c|c|}
\hline term description & $\begin{array}{l}\text { observed } \\
\text { gene count }\end{array}$ & $\begin{array}{l}\text { background } \\
\text { gene count }\end{array}$ & FDR & matching proteins in network (labels) \\
\hline response to bacterium & 10 & 555 & 1.17E-08 & $\begin{array}{l}\text { TGFB1,SERPINE1,CRP,VCAM1,TNFRSF11B,FGA,SELE,MBL } \\
2, \text { IL6,TNF }\end{array}$ \\
\hline $\begin{array}{l}\text { extracellular structure } \\
\text { organization }\end{array}$ & 9 & 339 & 1.17E-08 & $\begin{array}{l}\text { TGFB1,SERPINE1,VWF,PLA2G7,VCAM1,TNFRSF11B,FGA, } \\
\text { LPA,TNF }\end{array}$ \\
\hline inflammatory response & 9 & 482 & 8.37E-08 & TGFB1,IL5,CRP,VCAM1,TNFRSF11B,SELE,MBL2,IL6,TNF \\
\hline $\begin{array}{l}\text { regulation of inflammatory } \\
\text { response }\end{array}$ & 8 & 338 & $1.42 \mathrm{E}-07$ & SERPINE1,PROC,FABP4,PLA2G7,SELE,PROS1,IL6,TNF \\
\hline defense response & 11 & 1234 & 4.79E-07 & $\begin{array}{l}\text { TGFB1,SERPINE1,IL5,CRP,VCAM1,TNFRSF11B,FGA,SELE, } \\
\text { MBL2,IL6,TNF }\end{array}$ \\
\hline regulation of defense response & 9 & 676 & 7.10E-07 & $\begin{array}{l}\text { SERPINE1,PROC,FABP4,PLA2G7,FGA,SELE,PROS1,IL6,TN } \\
\text { F }\end{array}$ \\
\hline $\begin{array}{l}\text { regulation of response to } \\
\text { external stimulus }\end{array}$ & 10 & 955 & 7.10E-07 & $\begin{array}{l}\text { TGFB1,SERPINE1,PROC,FABP4,PLA2G7,FGA,SELE,PROS1, } \\
\text { IL6,TNF }\end{array}$ \\
\hline $\begin{array}{l}\text { extracellular matrix } \\
\text { organization }\end{array}$ & 7 & 296 & $1.18 \mathrm{E}-06$ & TGFB1,SERPINE1,VWF,VCAM1,TNFRSF11B,FGA,TNF \\
\hline response to lipopolysaccharide & 7 & 298 & $1.18 \mathrm{E}-06$ & TGFB1,SERPINE1,VCAM1,TNFRSF11B,SELE,IL6,TNF \\
\hline $\begin{array}{l}\text { positive regulation of } \\
\text { mononuclear cell migration }\end{array}$ & 4 & 25 & $1.84 \mathrm{E}-06$ & TGFB1,SERPINE1,PLA2G7,TNF \\
\hline response to stress & 14 & 3267 & $2.58 \mathrm{E}-06$ & $\begin{array}{l}\text { TGFB1,SERPINE1,IL5,PROC,CRP,VWF, VCAM1,TNFRSF11B } \\
\text {,FGA,SELE,MBL2,PROS1,IL6,TNF }\end{array}$ \\
\hline $\begin{array}{l}\text { positive regulation of immune } \\
\text { system process }\end{array}$ & 9 & 882 & $2.92 \mathrm{E}-06$ & TGFB1,SERPINE1,IL5,PLA2G7,VCAM1,FGA,MBL2,IL6,TNF \\
\hline immune system process & 12 & 2370 & $7.81 \mathrm{E}-06$ & $\begin{array}{l}\text { TGFB1,IL5,CRP,FTH1,VCAM1,TNFRSF11B,FGA,SELE,MBL } \\
\text { 2,PROS1,IL6,TNF }\end{array}$ \\
\hline $\begin{array}{l}\text { regulation of immune system } \\
\text { process }\end{array}$ & 10 & 1391 & $7.81 \mathrm{E}-06$ & $\begin{array}{l}\text { TGFB1,SERPINE1,IL5,PLA2G7,VCAM1,FGA,MBL2,PROS1,I } \\
\text { L6,TNF }\end{array}$ \\
\hline defense response to bacterium & 6 & 250 & $7.81 \mathrm{E}-06$ & SERPINE1,CRP,FGA,MBL2,IL6,TNF \\
\hline $\begin{array}{l}\text { positive regulation of } \\
\text { inflammatory response }\end{array}$ & 5 & 120 & $7.81 \mathrm{E}-06$ & SERPINE1,FABP4,PLA2G7,IL6,TNF \\
\hline $\begin{array}{l}\text { positive regulation of leukocyte } \\
\text { migration }\end{array}$ & 5 & 127 & 8.11E-06 & TGFB1,SERPINE1,PLA2G7,IL6,TNF \\
\hline platelet degranulation & 5 & 129 & 8.37E-06 & TGFB1,SERPINE1,VWF,FGA,PROS1 \\
\hline
\end{tabular}




\begin{tabular}{|c|c|c|c|c|}
\hline $\begin{array}{l}\text { negative regulation of blood } \\
\text { coagulation }\end{array}$ & 4 & 48 & $9.50 \mathrm{E}-06$ & SERPINE1,PROC,FGA,PROS1 \\
\hline $\begin{array}{l}\text { positive regulation of response } \\
\text { to external stimulus }\end{array}$ & 7 & 499 & $1.18 \mathrm{E}-05$ & TGFB1,SERPINE1,FABP4,PLA2G7,FGA,IL6,TNF \\
\hline $\begin{array}{l}\text { regulation of response to } \\
\text { stimulus }\end{array}$ & 14 & 3882 & $1.25 \mathrm{E}-05$ & $\begin{array}{l}\text { TGFB1,SERPINE1,IL5,PROC,FABP4,PLA2G7,VCAM1,TNFR } \\
\text { SF11B,FGA,SELE,MBL2,PROS1,IL6,TNF }\end{array}$ \\
\hline leukocyte migration & 6 & 296 & $1.25 \mathrm{E}-05$ & TGFB1,VCAM1,SELE,PROS1,IL6,TNF \\
\hline $\begin{array}{l}\text { regulation of immunoglobulin } \\
\text { production }\end{array}$ & 4 & 62 & 2.01E-05 & TGFB1,IL5,IL6,TNF \\
\hline $\begin{array}{l}\text { defense response to other } \\
\text { organism }\end{array}$ & 8 & 859 & $2.35 \mathrm{E}-05$ & TGFB1,SERPINE1,CRP,VCAM1,FGA,MBL2,IL6,TNF \\
\hline positive regulation of transport & 8 & 892 & $2.85 \mathrm{E}-05$ & TGFB1,SERPINE1,IL5,FGA,SELE,MBL2,IL6, TNF \\
\hline acute inflammatory response & 4 & 73 & $3.21 \mathrm{E}-05$ & CRP,VCAM1,MBL2,IL6 \\
\hline $\begin{array}{l}\text { positive regulation of defense } \\
\text { response }\end{array}$ & 6 & 365 & 3.27E-05 & SERPINE1,FABP4,PLA2G7,FGA,IL6,TNF \\
\hline $\begin{array}{l}\text { negative regulation of lipid } \\
\text { storage }\end{array}$ & 3 & 17 & $3.38 \mathrm{E}-05$ & CRP,IL6,TNF \\
\hline leukocyte tethering or rolling & 3 & 17 & $3.38 \mathrm{E}-05$ & VCAM1,SELE,TNF \\
\hline $\begin{array}{l}\text { regulation of immunoglobulin } \\
\text { secretion }\end{array}$ & 3 & 17 & $3.38 \mathrm{E}-05$ & IL5,IL6,TNF \\
\hline localization & 15 & 5233 & $3.46 \mathrm{E}-05$ & $\begin{array}{l}\text { TGFB1,SERPINE1,PROC,CRP,FABP4,VWF,FTH1,VCAM1,F } \\
\text { GA,LPA,SELE,MBL2,PROS1,IL6,TNF }\end{array}$ \\
\hline fibrinolysis & 3 & 21 & $4.81 \mathrm{E}-05$ & SERPINE1,FGA,PROS1 \\
\hline $\begin{array}{l}\text { response to tumor necrosis } \\
\text { factor }\end{array}$ & 5 & 217 & $4.91 \mathrm{E}-05$ & FABP4,VCAM1,TNFRSF11B,SELE,TNF \\
\hline regulation of protein secretion & 6 & 422 & 5.53E-05 & TGFB1,IL5,CRP,FGA,IL6,TNF \\
\hline response to cytokine & 8 & 1035 & 5.92E-05 & TGFB1,IL5,FABP4,VCAM1,TNFRSF11B,SELE,IL6,TNF \\
\hline regulation of endocytosis & 5 & 229 & 5.99E-05 & TGFB1,SERPINE1,SELE,MBL2,TNF \\
\hline $\begin{array}{l}\text { positive regulation of cell-cell } \\
\text { adhesion }\end{array}$ & 5 & 238 & 7.07E-05 & TGFB1,VCAM1,FGA,IL6,TNF \\
\hline $\begin{array}{l}\text { positive regulation of protein } \\
\text { secretion }\end{array}$ & 5 & 240 & $7.22 \mathrm{E}-05$ & TGFB1,IL5,FGA,IL6,TNF \\
\hline
\end{tabular}




\begin{tabular}{|c|c|c|c|c|}
\hline $\begin{array}{l}\text { negative regulation of protein } \\
\text { metabolic process }\end{array}$ & 8 & 1075 & 7.28E-05 & TGFB1,SERPINE1,FABP4,FGA,LPA,PROS1,IL6,TNF \\
\hline wound healing & 6 & 461 & 8.02E-05 & TGFB1,PROC,VWF,FGA,PROS1,IL6 \\
\hline $\begin{array}{l}\text { negative regulation of } \\
\text { multicellular organismal } \\
\text { process }\end{array}$ & 8 & 1098 & $8.08 \mathrm{E}-05$ & TGFB1,SERPINE1,PROC,TNFRSF11B,FGA,PROS1,IL6,TNF \\
\hline immune response & 9 & 1560 & $9.69 \mathrm{E}-05$ & TGFB1,IL5,FTH1,VCAM1,TNFRSF11B,FGA,MBL2,IL6,TNF \\
\hline $\begin{array}{l}\text { defense response to Gram- } \\
\text { positive bacterium }\end{array}$ & 4 & 111 & 9.69E-05 & CRP,MBL2,IL6,TNF \\
\hline regulation of body fluid levels & 6 & 483 & $9.69 \mathrm{E}-05$ & SERPINE1,PROC,VWF,FGA,PROS1,IL6 \\
\hline $\begin{array}{l}\text { regulation of vesicle-mediated } \\
\text { transport }\end{array}$ & 6 & 480 & $9.69 \mathrm{E}-05$ & TGFB1,SERPINE1,FGA,SELE,MBL2,TNF \\
\hline $\begin{array}{l}\text { regulation of superoxide } \\
\text { metabolic process }\end{array}$ & 3 & 33 & 0.00012 & TGFB1,CRP,TNF \\
\hline transport & 13 & 4130 & 0.00013 & $\begin{array}{l}\text { TGFB1,SERPINE1,PROC,CRP,FABP4,VWF,FTH1,FGA,LPA, } \\
\text { MBL2,PROS1,IL6,TNF }\end{array}$ \\
\hline blood coagulation & 5 & 288 & 0.00013 & PROC,VWF,FGA,PROS1,IL6 \\
\hline $\begin{array}{l}\text { positive regulation of } \\
\text { endocytosis }\end{array}$ & 4 & 129 & 0.00014 & SERPINE1,SELE,MBL2,TNF \\
\hline vesicle-mediated transport & 9 & 1699 & 0.00016 & $\begin{array}{l}\text { TGFB1,SERPINE1,PROC,CRP,VWF,FTH1,FGA,MBL2,PROS } \\
1\end{array}$ \\
\hline
\end{tabular}

\section{Blood biomarkers of stroke overlap with biomarkers of cellular senescence, thromboembolism and sarcopenia, with links to cancer}

Given the classification of the biomarkers we presented, it is of interest whether alternative classifications, relating to other biological processes, may be possible. On one hand, the GO annotations in Table 2 further refer to lipid storage (supported only by immuno-inflammatory proteins, though), protein secretion / endocytosis, wound healing, and some other general metabolic processes. On the other hand, we may consider more general biological processes such as sarcopenia, and overlapping biological processes such as cellular senescence (Mankhong et al., 2020). The latter is not frequently mentioned in the literature on predicting ischemic stroke outcome, though there are exceptions (Childs et al., 2017; Valenzuela et al., 2017). Our analysis corroborates the evidence for its role in the natural history of stroke-related health deterioration, given that many members of the SASP (senescence-associated secretory 
phenotype; SERPINE1, IL6, CRP, TNF and TGFB1) are placed center stage in the interaction network. At the minimum, we suggest that the inflammatory processes taking place after stroke are expected to be pushed further by senescent cells secreting just the factors that predict health deterioration after stroke. As we suggested elsewhere, cellular senescence may indeed be a driver of the co-morbidity of stroke and (pancreatic) cancer, including thromboembolic events (Henze et al., 2020).

In fact, thromboembolism biomarkers in patients with cancer were reviewed recently (Gervaso et al., 2021) and apart from platelet and leukocyte counts, the authors list tissue factor (Coagulation Factor III), d-dimers, soluble P-selectin and CRP, overlapping with the markers of Table 1. While these biomarkers refer to venous thromboembolism, data on biomarkers for arterial thrombotic events specifically in cancer patients are sparse (Gervaso et al., 2021). Very recently, a prospective study described biomarkers in cancer patients with stroke (not necessarily predictive of future outcomes), in stroke patients that do not feature cancer as a comorbidity, and it also contrasts these with another group of patients that are only affected by cancer (Navi et al., 2021); each patient group had size 50. Interestingly, most association biomarkers are valid for stroke patients no matter whether they are also affected by cancer; these are d-dimer, ICAM-1, VCAM-1, thrombomodulin. Only one biomarker (P-selectin) is found associated exclusively in the stroke-only group, and thrombin-antithrombin is found associated exclusively in the cancer-only group. Here, biomarkers were measured around $96 \mathrm{~h}$ after the event/diagnosis; multivariable linear regression was used to reveal associations to an outcome defined by prespecified hematological biomarkers and transcranial Doppler microemboli detection, adjusted for race, number of stroke risk factors, smoking, antithrombotics use, and NIHSS. Of interest, all biomarkers of Navi et al. (2021) are related to coagulation or cell adhesion, and we also note the overlap with the biomarkers of Table 1.

Finally, the discrimination between 100 sarcopenia patients and 100 controls was recently demonstrated by Marzetti et al. (2019), based on the proteins MPO, P-selectin, IL8, MCP-1, CRP, MIP-1 $\alpha$, PDGF-BB, and IP-10. Again, there is an overlap with our biomarkers, supporting the hypothesis that thrombotic events, including recurrent stroke, as well as sarcopenia may be triggered by post-stroke coagulation and inflammation. A recent transcriptomics analysis highlights the role of the aging immune system in acute stroke (Sykes et al., 2021) and suggests that not just the inflammatory aspects of the immune system play a role in determining disease outcome. However, the functional status of the (adaptive) immune system is not as easy to measure as its contribution to inflammatory processes, explaining why no such markers are prominently reported as protein biomarkers in the blood. Moreover, immunity and inflammation are closely connected in general, and even more so in stroke, considering the immune-cell infiltration that is triggered by stroke.

As the major limitation of any study of this kind, we are limited to the small sets of biomarkers measured a-priori in any of the studies performed thus far, resulting in inspector bias effects. Such a bias may be alleviated by high-throughput omics studies considering transcripts, proteins, lipids or other metabolites. However, transcripts are known to be quite noisy, largescale proteomics is lacking, and metabolites are not as straightforward in their assignment to biological processes, even though in this review, we consider some of these alongside the proteins. Nevertheless, the most recent transcriptomic analysis (Sykes et al., 2021) highlighted immune-related processes that are closely associated with the observations presented in this review, including interleukin signalling, though on the gene level, they found no transcripts that 
directly correspond to any of the protein biomarkers in Table 1.

Overall, we suggest that our summary of the outcome biomarkers for ischemic stroke provides strong evidence for the role of immune $\square$ inflammatory, coagulation-related, and adhesionrelated processes in the health deterioration and mortality after stroke. The overlap of the outcome biomarkers with the SASP and with thromboembolism biomarkers, as well as the role of senescent cells in blood clotting (Tanaka et al., 2018), suggests that cellular senescence plays a role as well. Cellular senescence is also implicated in sarcopenia (Mankhong et al., 2020), and we found an overlap of our outcome biomarkers for stroke with sarcopenia biomarkers, suggesting further that sarcopenia is a specific form of health deterioration that may also be co-driven by cellular senescence.

\section{Conflict of Interest:}

UW reports grants and personal fees from Merz Pharma, and personal fees from Allergan, Ipsen Pharma, Bristol-Myers Squibb, Daiichi Sankyo, Bayer Vital, Boehringer Ingelheim, Pfizer, Thieme, and Elsevier Press, all outside the submitted work. The other authors have nothing to disclose.

\section{Acknowledgements:}

We acknowledge the financial support by the Federal Ministry of Education and Research (BMBF) of Germany for the SASKit study (FKZ 01ZX1903A). The funder had no role in the design of the study.

\section{References}

Alfieri, D. F., Lehmann, M. F., Flauzino, T., de Araujo, M. C. M., Pivoto, N., Tirolla, R. M., Simao, A. N. C., Maes, M., Reiche, E. M. V., 2020. Immune-Inflammatory, Metabolic, Oxidative, and Nitrosative Stress Biomarkers Predict Acute Ischemic Stroke and ShortTerm Outcome. Neurotox Res 38, 330-343.

Childs, B. G., Gluscevic, M., Baker, D. J., Laberge, R. M., Marquess, D., Dananberg, J., van Deursen, J. M., 2017. Senescent cells: an emerging target for diseases of ageing. Nat Rev Drug Discov 16, 718-735.

Donkel, S. J., Benaddi, B., Dippel, D. W. J., Ten Cate, H., de Maat, M. P. M., 2019. Prognostic Hemostasis Biomarkers in Acute Ischemic Stroke. Arterioscler Thromb Vasc Biol 39, 360372.

Donkor, E. S., 2018. Stroke in the 21(st) Century: A Snapshot of the Burden, Epidemiology, and Quality of Life. Stroke Res Treat 2018, 3238165.

Draaisma, L. R., Wessel, M. J., Hummel, F. C., 2020. Neurotechnologies as tools for cognitive rehabilitation in stroke patients. Expert Rev Neurother 20, 1249-1261.

Friedmann, A. P., Koutychenko, A., Wu, C., Fredenburgh, J. C., Weitz, J. I., Gross, P. L., Xu, P., Ni, F., Kim, P. Y., 2019. Identification and characterization of a factor Va-binding site on human prothrombin fragment 2. Sci Rep 9, 2436. 
Fuellen, G., Liesenfeld, O., Kowald, A., Barrantes, I., Bastian, M., Simm, A., Jansen, L., TietzLatza, A., Quandt, D., Franceschi, C., Walter, M., 2020. The preventive strategy for pandemics in the elderly is to collect in advance samples \& data to counteract chronic inflammation (inflammaging). Ageing Res Rev, 101091.

Gamez, A., Alva, N., Carbonell, T., Rama, R., 2021. Extracellular ferritin contributes to neuronal injury in an in vitro model of ischemic stroke. J Physiol Biochem

Garcia-Yebenes, I., Sobrado, M., Moraga, A., Zarruk, J. G., Romera, V. G., Pradillo, J. M., Perez de la Ossa, N., Moro, M. A., Davalos, A., Lizasoain, I., 2012. Iron overload, measured as serum ferritin, increases brain damage induced by focal ischemia and early reperfusion. Neurochem Int 61, 1364-9.

Garg, R., Aravind, S., Kaur, S., Singh Chawla, S. P., Aggarwal, S., Goyal, G., 2020. Role of serum ferritin as a prognostic marker in acute ischemic stroke: A preliminary observation. Ann Afr Med 19, 95-102.

Gervaso, L., Dave, H., Khorana, A. A., 2021. Venous and Arterial Thromboembolism in Patients With Cancer: JACC: CardioOncology State-of-the-Art Review. JACC: CardioOncology 3, 173-190.

Hankey, G. J., 2014. Secondary stroke prevention. Lancet Neurol 13, 178-94.

Henze, L., Walter, U., Murua Escobar, H., Junghanss, C., Jaster, R., Kohling, R., Lange, F., Salehzadeh-Yazdi, A., Wolkenhauer, O., Hamed, M., Barrantes, I., Palmer, D., Moller, S., Kowald, A., Heussen, N., Fuellen, G., 2020. Towards biomarkers for outcomes after pancreatic ductal adenocarcinoma and ischaemic stroke, with focus on (co)-morbidity and ageing/cellular senescence (SASKit): protocol for a prospective cohort study. BMJ Open 10, e039560.

Hisada, Y., Mackman, N., 2017. Cancer-associated pathways and biomarkers of venous thrombosis. Blood 130, 1499-1506.

Jensen, L. J., Kuhn, M., Stark, M., Chaffron, S., Creevey, C., Muller, J., Doerks, T., Julien, P., Roth, A., Simonovic, M., Bork, P., von Mering, C., 2009. STRING 8--a global view on proteins and their functional interactions in 630 organisms. Nucleic Acids Res 37, D412-6.

Lai, Y. J., Hanneman, S. K., Casarez, R. L., Wang, J., McCullough, L. D., 2019. Blood biomarkers for physical recovery in ischemic stroke: a systematic review. Am J Transl Res 11, 4603-4613.

Lehmann, A., Alfieri, D. F., de Araujo, M. C. M., Trevisani, E. R., Nagao, M. R., Pesente, F. S., Gelinski, J. R., de Freitas, L. B., Flauzino, T., Lehmann, M. F., Lozovoy, M. A. B., Bregano, J. W., Simao, A. N. C., Maes, M., Reiche, E. M. V., 2021. Immune-inflammatory, coagulation, adhesion, and imaging biomarkers combined in machine learning models improve the prediction of death 1 year after ischemic stroke. Clin Exp Med

Li, X., Lin, S., Chen, X., Huang, W., Li, Q., Zhang, H., Chen, X., Yang, S., Jin, K., Shao, B., 2019. The Prognostic Value of Serum Cytokines in Patients with Acute Ischemic Stroke. Aging Dis 10, 544-556.

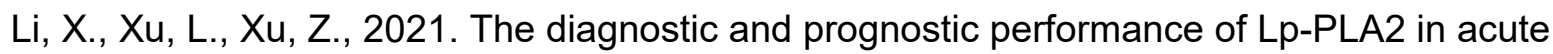
ischemic stroke. Med Clin (Barc) 156, 437-443.

Mankhong, S., Kim, S., Moon, S., Kwak, H. B., Park, D. H., Kang, J. H., 2020. Experimental Models of Sarcopenia: Bridging Molecular Mechanism and Therapeutic Strategy. Cells 9, 1385.

Marzetti, E., Picca, A., Marini, F., Biancolillo, A., Coelho-Junior, H. J., Gervasoni, J., Bossola, M., Cesari, M., Onder, G., Landi, F., Bernabei, R., Calvani, R., 2019. Inflammatory signatures in older persons with physical frailty and sarcopenia: The frailty "cytokinome" at its core. Exp Gerontol 122, 129-138. 
Millan, M., Sobrino, T., Arenillas, J. F., Rodriguez-Yanez, M., Garcia, M., Nombela, F., Castellanos, M., de la Ossa, N. P., Cuadras, P., Serena, J., Castillo, J., Davalos, A., 2008. Biological signatures of brain damage associated with high serum ferritin levels in patients with acute ischemic stroke and thrombolytic treatment. Dis Markers 25, 181-8.

Millan, M., Sobrino, T., Castellanos, M., Nombela, F., Arenillas, J. F., Riva, E., Cristobo, I., Garcia, M. M., Vivancos, J., Serena, J., Moro, M. A., Castillo, J., Davalos, A., 2007. Increased body iron stores are associated with poor outcome after thrombolytic treatment in acute stroke. Stroke 38, 90-5.

Montellano, F. A., Ungethum, K., Ramiro, L., Nacu, A., Hellwig, S., Fluri, F., Whiteley, W. N., Bustamante, A., Montaner, J., Heuschmann, P. U., 2021. Role of Blood-Based Biomarkers in Ischemic Stroke Prognosis: A Systematic Review. Stroke 52, 543-551.

Navi, B. B., Sherman, C. P., Genova, R., Mathias, R., Lansdale, K. N., LeMoss, N. M., Wolfe, J., Skakodub, A., Kamel, H., Tagawa, S. T., Saxena, A., Ocean, A. J., Soff, G. A., DeSancho, M. T., ladecola, C., Elkind, M. S. V., Peerschke, E., Zhang, C., DeAngelis, L. M., 2021. Mechanisms of Ischemic Stroke in Patients with Cancer: A Prospective Study. Ann Neurol 90, 159-169.

O'Donnell, M. J., Chin, S. L., Rangarajan, S., Xavier, D., Liu, L., Zhang, H., Rao-Melacini, P., Zhang, X., Pais, P., Agapay, S., Lopez-Jaramillo, P., Damasceno, A., Langhorne, P., McQueen, M. J., Rosengren, A., Dehghan, M., Hankey, G. J., Dans, A. L., Elsayed, A., Avezum, A., Mondo, C., Diener, H. C., Ryglewicz, D., Czlonkowska, A., Pogosova, N., Weimar, C., Iqbal, R., Diaz, R., Yusoff, K., Yusufali, A., Oguz, A., Wang, X., Penaherrera, E., Lanas, F., Ogah, O. S., Ogunniyi, A., Iversen, H. K., Malaga, G., Rumboldt, Z., Oveisgharan, S., Al Hussain, F., Magazi, D., Nilanont, Y., Ferguson, J., Pare, G., Yusuf, S., investigators, I., 2016. Global and regional effects of potentially modifiable risk factors associated with acute stroke in 32 countries (INTERSTROKE): a case-control study. Lancet 388, 761-75.

Reiche, E. M. V., Gelinksi, J. R., Alfieri, D. F., Flauzino, T., Lehmann, M. F., de Araujo, M. C. M., Lozovoy, M. A. B., Simao, A. N. C., de Almeida, E. R. D., Maes, M., 2019. Immuneinflammatory, oxidative stress and biochemical biomarkers predict short-term acute ischemic stroke death. Metab Brain Dis 34, 789-804.

Soriano-Tarraga, C., Lazcano, U., Jimenez-Conde, J., Ois, A., Cuadrado-Godia, E., GiraltSteinhauer, E., Rodriguez-Campello, A., Gomez-Gonzalez, A., Avellaneda-Gomez, C., Vivanco-Hidalgo, R. M., Roquer, J., 2021. Biological age is a novel biomarker to predict stroke recurrence. J Neurol 268, 285-292.

Sykes, G. P., Kamtchum-Tatuene, J., Falcione, S., Zehnder, S., Munsterman, D., Stamova, B., Ander, B. P., Sharp, F. R., Jickling, G., 2021. Aging Immune System in Acute Ischemic Stroke: A Transcriptomic Analysis. Stroke 52, 1355-1361.

Tanaka, T., Biancotto, A., Moaddel, R., Moore, A. Z., Gonzalez-Freire, M., Aon, M. A., Candia, J., Zhang, P., Cheung, F., Fantoni, G., consortium, C. H. I., Semba, R. D., Ferrucci, L., 2018. Plasma proteomic signature of age in healthy humans. Aging Cell 17, e12799.

Uivarosan, D., Bungau, S., Tit, D. M., Moisa, C., Fratila, O., Rus, M., Bratu, O. G., Diaconu, C. C., Pantis, C., 2020. Financial Burden of Stroke Reflected in a Pilot Center for the Implementation of Thrombolysis. Medicina (Kaunas) 56

Valenzuela, C. A., Quintanilla, R., Moore-Carrasco, R., Brown, N. E., 2017. The Potential Role of Senescence As a Modulator of Platelets and Tumorigenesis. Front Oncol 7, 188.

Zauli, G., Corallini, F., Bossi, F., Fischetti, F., Durigutto, P., Celeghini, C., Tedesco, F., Secchiero, P., 2007. Osteoprotegerin increases leukocyte adhesion to endothelial cells both in vitro and in vivo. Blood 110, 536-43. 